\title{
Cargo por confiabilidad: ¿éxito o fracaso? ${ }^{1}$
}

\author{
ANDRÉS DAVID OSPINA RIAÑO2 \\ DIEWISKEY MOSQUERA PALACIOS ${ }^{3}$
}

\section{RESUMEN}

Con el objetivo de garantizar la confiabilidad en el suministro de energía eléctrica para el país, la Comisión de Regulación de Energía y Gas (CREG) diseñó, en el año $2006^{4}$, el esquema del cargo por confiabilidad.

Dicho esquema está compuesto por: (i) una remuneración que se paga por la disponibilidad futura de los activos de generación que requiere el país, y que pretende garantizar la entrega de la energía comprometida por los beneficiarios del cargo, previa asignación de una serie de cantidades de energía para entregar a futuro por estos, a través de un mecanismo de subasta, y (ii) un precio de escasez que establece la autoridad regulatoria, y que activa la exigibilidad de las obligaciones de energía comprometidas, cuando el precio de la energía en la bolsa supera el citado precio de escasez.

Este mecanismo de remuneración de obligaciones futuras de entrega de energía ha viabilizado la inversión en los recursos de generación que requiere el país, para garantizar de manera eficiente la atención de la demanda de energía en condiciones críticas de abastecimiento, brindando señales económicas de inversión a largo plazo y permitiendo la estabilización de los ingresos de los generadores beneficiarios del cargo ${ }^{5}$.

No obstante los citados beneficios, y con ocasión del fenómeno de El Niño que ha afectado al país y de algunas situaciones de presunto incumplimiento en la entrega de las obligaciones de energía firme a cargo de un número reducido de beneficiarios del

1 Para citar el artículo: OSPINA, A. y MOSQUERA, D. Cargo por confiabilidad: ¿Éxito o fracaso?, en: Revista Con-texto, n. ${ }^{\circ} 45$, pp. 13-36. DOI: https://doi.org/10.18601/01236458.n45.03

2 Magíster en Derecho Económico Universidad Externado de Colombia, Director Jurídico Empresa de Generación y Promoción de Energía de Antioquía. Correo-e: andresda2002@yahoo.es

3 Magíster en Derecho Económico Universidad Externado de Colombia, Gerente QM+R Asesores. Correo-e: diewiskey@yahoo.es

4 Colombia, Comisión de Regulación de Energía y Gas - CREG. Resolución CREG 071 de 2006 (3 de octubre de 2006), por la cual se adopta la metodología para la remuneración del cargo por confiabilidad en el Mercado de Energía Mayorista. Publicada en el Diario Oficial no. 46.421 el día 16 de octubre de 2006.

5 UPME, Plan de Expansión de Generación y Transmisión 2015-2029, p. 119. Disponible en Internet: http://www.upme.gov.co/Docs/Plan_Expansion/2016/Plan_Expansion_GT_2015-2029/Plan_GT_20152029_VF_22-12-2015.pdf 
cargo $^{6}$, han surgido voces críticas en relación con el esquema del cargo por confiabilidad, que obligan a la comunidad jurídica a analizar la situación por la que atraviesa el Mercado de Energía Mayorista colombiano (MEM), así como la eficiencia del mecanismo diseñado por el regulador.

Principalmente, surge una serie de inquietudes que pueden resumirse en cuatro básicas, que serán desarrolladas en el presente documento, así: (i) cuál es la naturaleza jurídica y económica del cargo por confiabilidad, (ii) si dicha herramienta genera obligaciones de disponibilidad para los agentes que reciben asignaciones de obligaciones de energía firme, (iii) si el diseño estructural de la metodología del cargo por confiabilidad debe ser revisado y por qué y (iv) qué soluciones podrían plantearse desde lo jurídico, no solo para mejorar la estructura de la metodología bajo análisis, sino para atender de manera integral la crítica situación por la que atraviesa el Sistema Interconectado Nacional (SIN).

Palabras Claves: servicios públicos, cargo por confiabilidad, obligaciones de energía firme, opciones financieras.

\section{RELIABILITY COST: SUCCESS OR FAILURE?}

\section{ABSTRACT}

In order to ensure reliable supply of electricity for the country, the CREG designed, in 2006, the scheme of charge for reliability.

This scheme, consists of (i) remuneration paid for the present and future availability of generation assets required by the country, and (ii) a scarcity price established by the regulatory authority, and that activates the fulfillment of obligations of committed energy when the price of energy in the bag exceeds the scarcity price.

This scheme is successful, but some problems arising from the El Niño phenomenon and some situations of failure to deliver firm energy obligations by a small number of beneficiaries of the mechanism, have resulted in critical voices regarding the scheme of reliability charge.

Keywords: public utilities, reliability charge, firm energy obligations, financial options.

6 Redacción Economía. "Termocandelaria no generó energía pese a que su planta estaba disponible. El Espectador [en línea], 12 de noviembre de 2015. Disponible en Internet: http://www.elespectador.com/ noticias/economia/superservicios-abre-investigacion-contra-termocandelari-articulo-598727 y Unidad Investigativa", en Termocandelaria tienen empeñadas basta las turbinas. El Tiempo [en línea], 20 de noviembre de 2015. Disponible en Internet: http://www.eltiempo.com/economia/empresas/termocandelaria-tienenempenadas-hasta-las-turbinas/16435992 
1. LA CONFIABILIDAD EN EL SUMINISTRO DE ENERGÍA ELÉCTRICA: PRINCIPIO BÁSICO DEL ORDENAMIENTO JURÍDICO "ELÉCTRICO" COLOMBIANO

De conformidad con lo dispuesto en el artículo 365 de nuestra actual Constitución Política $^{7}$ : (i) los servicios públicos son inherentes a la finalidad social del Estado, por lo que (ii) es deber de este asegurar la prestación eficiente de aquellos, a todos los habitantes del territorio nacional.

Lo anterior, por cuanto se considera que los servicios públicos, y en especial aquellos que se enmarcan dentro de la categoría de domiciliarios, son un instrumento necesario para la satisfacción plena de las necesidades básicas que todas las personas, sin excepción, demandan. De lo anterior se desprende que sin la prestación continua y eficiente de dichos servicios, no puede pretenderse la proyección de una sociedad hacia su desarrollo, ni mucho menos el cumplimiento de los fines de un Estado Social de Derecho como el nuestro.

Vale la pena anotar que en Colombia, a partir de la expedición de la Constitución de 1991, el modelo jurídico adoptado privilegia la prestación de tales servicios por terceros, en pro de una mayor eficiencia, reservándose el Estado las funciones de regulación, y de inspección, control y vigilancia, y reconociendo a los empresarios la recuperación de los costos involucrados en la construcción y operación de los activos y redes humanas y físicas que para la prestación se requieren.

Dicho modelo ha sido exitoso y ha permitido, en el caso del sector eléctrico, la construcción de un mercado sólido y moderno, el aumento de coberturas de prestación ${ }^{8}$, una mejora ostensible de la calidad y continuidad del servicio y la proyección del país como exportador de energía eléctrica en el hemisferio.

Teniendo en cuenta los citados postulados constitucionales, la Ley 142 de 1994 estatuye en el numeral 3 de su artículo $3^{[9]}$ que uno de los objetivos de la intervención del Estado en los servicios públicos es fijar metas de eficiencia, cobertura y calidad, de manera tal que se garantice una prestación eficiente, continua e ininterrumpida de estos.

Por su parte, el artículo 4 de la Ley 143 de $1994^{[10]}$ indica que el Estado, en relación con el servicio de energía, tendrá como objetivos, en el cumplimiento de sus funciones, los de (i) abastecer la demanda de electricidad de la comunidad bajo criterios económicos y de viabilidad financiera, asegurando su cubrimiento en un marco de uso racional

7 Colombia, Asamblea Nacional Constituyente. Artículo 365, Constitución Política de Colombia (20 de julio de 1991). Gaceta Constitucional. Bogotá, 1991, n. ${ }^{\circ} 116$.

8 Contraloría General de la República. Colombia: Entre la exclusión y el desarrollo - Propuestas para la transición al Estado Social del Derecho. Bogotá: CGR, 2012, p. 238.

9 Colombia, Congreso de la República. Artículo 3, Ley 142 de 1994 (11 de julio de 1994). Por la cual se establece el régimen de los servicios públicos domiciliarios y se dictan otras disposiciones. Diario Oficial. Bogotá, 1994, n. ${ }^{\circ} 41.433$.

10 Colombia, Congreso de la República. Artículo 4, Ley 143 de 1994 (12 de julio de 1994). Por la cual se establece el régimen para la generación, interconexión, transmisión, distribución y comercialización de electricidad en el territorio nacional, se conceden unas autorizaciones y se dictan otras disposiciones en materia energética. Diario Oficial. Bogotá, 1994, n. ${ }^{\circ} 41.434$. 
y eficiente de los diferentes recursos energéticos del país, y (ii) asegurar una operación eficiente, segura y confiable en las actividades del sector, manteniendo los niveles de calidad y seguridad establecidos.

Lo anterior concuerda con lo dispuesto en el artículo 20 de la citada Ley 143 de $1994^{[11]}$, el cual definió como objetivo fundamental de la regulación en el sector eléctrico el de asegurar una adecuada prestación del servicio mediante el aprovechamiento eficiente de los diferentes recursos energéticos, en beneficio del usuario, en términos de calidad, oportunidad y costo del servicio.

Ahora bien, para el cumplimiento del objetivo señalado, el artículo 23 de la Ley 143 de $1994^{[12]}$ le atribuyó a la Comisión de Regulación de Energía y Gas, entre otras, las funciones de: (i) crear las condiciones para asegurar la disponibilidad de una oferta energética eficiente capaz de abastecer la demanda bajo criterios sociales, económicos, ambientales y de viabilidad financiera, promover y preservar la competencia, para lo cual, la oferta eficiente, en el sector eléctrico, debe tener en cuenta la capacidad de generación de respaldo; (ii) valorar la capacidad de generación de respaldo de la oferta eficiente $_{i}$ (iii) definir y hacer operativos los criterios técnicos de calidad, confiabilidad y seguridad del servicio de energía; (iv) establecer el Reglamento de Operación para realizar el planeamiento y la coordinación de la operación del Sistema Interconectado Nacional, y (v) determinar las condiciones para la liberación gradual del mercado hacia la libre competencia.

Las funciones antes señaladas concuerdan con las establecidas en el artículo 74 de la Ley 142 de $1994^{[13]}$, que indica que son funciones y facultades especiales de la CREG, entre otras, regular el ejercicio de las actividades de los sectores de energía y gas combustible, para asegurar la disponibilidad de una oferta energética eficiente ; propiciar la competencia en el sector de minas y energía y proponer la adopción de las medidas necesarias para impedir abusos de posición dominante; buscar la liberación gradual de los mercados hacia la libre competencia, y establecer criterios para la fijación de compromisos de ventas garantizadas de energía y potencia entre las empresas eléctricas y entre estas y los grandes usuarios.

De acuerdo con lo expuesto, se observa cómo desde el marco constitucional y legal, se ha establecido la confiabilidad en el suministro de energía como una meta fundamental

11 Colombia, Congreso de la República. Artículo 20, Ley 143 de 1994 (12 de julio de 1994). Por la cual se establece el régimen para la generación, interconexión, transmisión, distribución y comercialización de electricidad en el territorio nacional, se conceden unas autorizaciones y se dictan otras disposiciones en materia energética. Diario Oficial. Bogotá, 1994, n. ${ }^{\circ} 41.434$.

12 Colombia, Congreso de la República. Artículo 23, Ley 143 de 1994 (12 de julio de 1994). Por la cual se establece el régimen para la generación, interconexión, transmisión, distribución y comercialización de electricidad en el territorio nacional, se conceden unas autorizaciones y se dictan otras disposiciones en materia energética. Diario Oficial. Bogotá, 1994, n. ${ }^{\circ} 41.434$.

13 Colombia, Congreso de la República. Artículo 74, Ley 142 de 1994 (11 de julio de 1994). Por la cual se establece el régimen de los servicios públicos domiciliarios y se dictan otras disposiciones. Diario Oficial. Bogotá, 1994, n. ${ }^{\circ} 41.433$. 
de la regulación sectorial, la cual debe cumplirse bajo el marco del principio de libertad de entrada y de salida de los inversionistas en el sector.

Dado lo anterior, en el presente documento se analizará el principal de los mecanismos diseñado por el regulador para garantizar la confiabilidad en el suministro eléctrico, el cual se denomina cargo por confiabilidad.

\section{CARGO POR CONFIABILIDAD - NATURALEZA JURÍDICA}

Para iniciar el presente título, lo primero que ha de decirse es que la actual metodología para la remuneración del cargo por confiabilidad en el Mercado de Energía Mayorista fue adoptada en la Resolución CREG 071 de $2006^{[14]}$, con la cual se reemplazó la metodología del cargo por capacidad ${ }^{15}$ que rigió en Colombia desde el $1 .{ }^{\circ}$ de diciembre de 1996 y hasta el 30 de noviembre de 2006.

La nueva metodología adoptada por la CREG contiene una serie de herramientas dirigidas a: (i) incentivar las inversiones que se requieran en el sector eléctrico, para garantizar la expansión de la capacidad instalada de generación que demanda el Sistema Interconectado Nacional (SIN), y, a través de dicho objetivo, (ii) lograr garantizar la prestación de un servicio de energía de calidad, en condiciones de continuidad y confiabilidad aceptables.

Dicho lo anterior, y previo a analizar la naturaleza jurídica de la metodología del cargo por confiabilidad, es menester indicar que si bien dicha metodología busca el incentivo a las inversiones en expansión del parque generador, lo cierto es que no se obliga a los inversionistas a desarrollar proyectos, pues en la regulación eléctrica colombiana no está prevista la posibilidad legal de que el Estado celebre contratos con inversionistas para que estos los adelanten ${ }^{16}$.

Teniendo en cuenta lo expuesto, se tiene que la regla general será la ejecución de proyectos de generación por parte del sector privado, bajo la premisa constitucional y legal de la libertad de entrada y salida, lo que asegura que dados unos incentivos ${ }^{17}$, los inversionistas puedan invertir o desinvertir en proyectos de generación o en cualquier

14 Colombia, Comisión de Regulación de Energía y Gas - CREG. Resolución CREG 071 de 2006 (3 de octubre de 2006). Por la cual se adopta la metodología para la remuneración del cargo por confiabilidad en el Mercado de Energía Mayorista. Publicada en el Diario Oficial n. ${ }^{\circ} 46.421$ el día 16 de octubre de 2006.

15 Colombia, Comisión de Regulación de Energía y Gas - CREG. Resolución CREG 116 de 1996 (28 de noviembre de 1996). Por la cual se precisa el método de cálculo del cargo por capacidad en el Mercado Mayorista de Electricidad y se aplaza su fecha de entrada en vigencia. Publicada en el Diario Oficial n. ${ }^{\circ}$ 42.929 el día 29 de noviembre de 1996.

16 Colombia, Congreso de la República. Artículo 10, Ley 142 de 1994 (11 de julio de 1994). Por la cual se establece el régimen de los servicios públicos domiciliarios y se dictan otras disposiciones. Diario Oficial. Bogotá, 1994, n. ${ }^{\circ} 41.433$.

17 Colombia, Congreso de la República. Artículo 3, Ley 142 de 1994 (11 de julio de 1994). Por la cual se establece el régimen de los servicios públicos domiciliarios y se dictan otras disposiciones. Diario Oficial. Bogotá, 1994, n. ${ }^{\circ} 41.433$. 
otro tipo de proyectos en otros sectores, de acuerdo a los análisis de rentabilidad y retorno que estos realicen.

Es por ello que, de entrada, debe indicarse que la metodología del cargo por confiabilidad carece de la naturaleza de un negocio jurídico unilateral, pues claramente la regulación de la CREG brinda un marco jurídico que promueve las inversiones sectoriales, bajo el entendido de que quien invierte en el sector se aviene en derechos y obligaciones a lo que la regulación estipule.

Al respecto de lo anterior, conviene tener en cuenta que las Leyes 142 y 143 de 1994 han facultado a la CREG para expedir normas jurídicas de carácter obligatorio para el sector eléctrico, a través de actos administrativos generales, que de manera alguna pueden ser entendidos como negocios jurídicos de naturaleza unilateral. En esa medida, debe observarse a la CREG como un regulador, y a los regulados como agentes libres que deciden invertir recursos en una actividad sujeta a la regulación y la vigilancia del Estado, asumiendo estos últimos los riesgos, derechos y responsabilidades inherentes a sus decisiones de negocios.

Volviendo al punto de análisis, debe recordarse que el artículo 23 de la Ley 143 de $1994^{[18]}$, o Ley Eléctrica, atribuyó a la CREG la función de "crear las condiciones para asegurar la disponibilidad de una oferta energética eficiente, capaz de abastecer la demanda bajo criterios sociales, económicos, ambientales y de viabilidad financiera, promover y preservar la competencia", así como la de "definir y bacer operativos los criterios técnicos de calidad, confiabilidad y seguridad del servicio de energía".

Bajo dicha premisa legal, la Comisión de Regulación, en el año 1996 ${ }^{[19]}$, puso en vigencia en el Mercado de Energía Mayorista, el mecanismo conocido como cargo por capacidad, que se pagó a la oferta eficiente que aportó firmeza al sistema bajo determinadas condiciones de hidrología crítica, hasta el año 2006, momento en el cual dicho instrumento fue reemplazado por el actual cargo por confiabilidad.

Valga la pena anotar que tal como lo expone la Compañía Expertos en Mercados S.A. E.S.P., XM S.A. E.S.P., que funge como administradora del mercado de energía ${ }^{20}$, $E l$ anterior modelo de Cargo por Capacidad consistía en un mecanismo de remuneración administrado de la capacidad de generación, que garantizaba un ingreso fijo anual por megavatio instalado, a un precio definido por el regulador", sin que existiera "... una obligación concreta de los generadores, asociada a esta remuneración".

Por su parte, el esquema del cargo por confiabilidad, implementado a través de la Resolución CREG 071 de 2006, no solo tiene en cuenta la capacidad instalada, sino que remunera una obligación de generar asociada a la infraestructura de generación que se tenga. En palabras de XM:

18 Colombia, Congreso de la República. Op. cit.

19 Colombia, Comisión de Regulación de Energía y Gas - CREG. Op. cit.

20 XM S.A. E.S.P. ABC del Cargo por Confiabilidad. Disponible en Internet: http://www.xm.com.co/Promocin\%20Primera \%20Subasta\%20de\%20Energa\%20Firme/abc2.pdf 
„El nuevo esquema de Cargo por Confiabilidad permite a los generadores contar con un ingreso de dinero fijo, independientemente de su participación diaria en el mercado mayorista, asignado para períodos de basta 20 años, reduciendo el riesgo de su inversión. Como contraprestación, los generadores deben mantenerse disponibles y cumplir con sus Obligaciones de Energía Firme cuando se presenten las condiciones de escasez en el sistema. Para la asignación y la determinación del precio se utiliza un mecanismo de mercado que propende por la eficiencia: las subastas de obligaciones de energía firme".

¿Pero cómo funciona dicho cargo? Pues bien, para explicar la forma como opera el cargo, ha de recordarse que las transacciones de energía en el Mercado de Energía Mayorista se efectúan bajo tres modalidades, así: i) contratos bilaterales financieros de compra de energía entre agentes generadores y comercializadores en cualquier combinación (G-C, G-G, C-C), ii) transacciones horarias directas en la bolsa de energía, definidas día a día, cuyos precios y cantidades físicas son determinados por las declaraciones de disponibilidad de recursos, y la interacción horaria entre oferta y demanda, de acuerdo a las condiciones establecidas en el Reglamento de Operación, y iii) subastas para la asignación de obligaciones de energía firme (OEF), del cargo por confiabilidad, cuyos precios y cantidades también son determinados por la interacción de la oferta y la demanda.

En relación con el tercero de los instrumentos citados, debe señalarse que introduce en el Mercado de Energía Mayorista un mercado de energía firme, con obligaciones que pueden tener una vigencia desde un año, hasta veinte años, según lo elija el inversionista para las plantas definidas como nuevas, y hasta de diez años, según la decisión de los agentes con plantas calificadas como especiales.

En este caso, y para cumplir los objetivos a que nos referimos anteriormente, se realizan asignaciones de cantidades de energía firme por los periodos antes anotados, a través de un mecanismo de subasta, de manera tal que los agentes generadores ganadores de la subasta adquieren un compromiso remunerado de generar determinadas cantidades de energía a un precio igualmente determinado en el momento en que sean llamados a despacho. Lo anterior de manera que se asegure la atención oportuna y eficiente de la demanda energética nacional, así como la remuneración de los inversionistas que libremente deciden participar en las subastas.

Al respecto de lo anterior, la doctrina ha definido el cargo por confiabilidad así ${ }^{21}$ :

„El Cargo por Confiabilidad implantado en el mercado colombiano sigue esta línea. En esencia, el mecanismo consiste en la compra, por parte del regulador en nombre del conjunto del sistema, de energía firme de largo plazo mediante subastas destinadas a potenciales inversores en nueva generación. En dicha licitación se pide a los ofertantes el precio, por un producto de largo plazo, la energía firme, que les garantiza una determinada remuneración durante un periodo de varios años a cambio de instalar un grupo generador y suplir la energía en los casos en que se exija».

21 Salazar, Jorge Enrique. Simulación del cargo por confiabilidad y de la expansión del mercado de generación en Colombia. Tesis de máster. Escuela Técnica Superior de Ingeniería - ICAI, 2008, p. 24. 
De esta manera, el cargo por confiabilidad se convierte en un mecanismo que busca incentivar y valorar la construcción de capacidad de respaldo que la oferta eficiente aporta al sistema, en cumplimiento de lo establecido en la Constitución Política y en las Leyes 142 y 143 de 1994.

Dicho cargo, de acuerdo con lo establecido en la Resolución CREG 071 de 2006, remunera la energía que está asociada a la capacidad de generación de respaldo de que trata el artículo 23 de la Ley 143 de 1994, y que es la que puede comprometerse para garantizar a los usuarios la confiabilidad en la prestación del servicio de energía eléctrica, bajo condiciones de estrés del Sistema Interconectado Nacional (SIN).

En consideración a lo expuesto, bien puede decirse que la naturaleza jurídica del cargo por confiabilidad es la de una opción financiera del tipo call en la que los generadores o inversionistas beneficiarios del cargo son remunerados para que, en el momento en que se haga efectiva la opción, entren a generar a un precio determinado. De esta manera, el cargo por confiabilidad representaría un pago anticipado de una capacidad de generación de respaldo, necesaria para atender situaciones críticas de suboferta energética.

Dicho lo anterior, y para precisar aún más la naturaleza de esta opción financiera de pago anticipado, conviene tener en cuenta las principales características de la metodología del cargo por confiabilidad:

- El cargo por confiabilidad busca valorar y remunerar la confiabilidad que aporta al sistema una determinada oferta energética, para lo cual se establecen unas obligaciones de energía firme (OEF), que se asignan a cada generador a través de un mecanismo de subasta, a un precio que es pagado por la demanda, independientemente de que se presenten o no las condiciones críticas que activen la necesidad de hacer exigibles las OEF. El mecanismo de asignación, que es la subasta de OEF, es un mecanismo transparente al que tienen acceso todos los inversionistas y/o agentes que cumplan con las condiciones establecidas por la regulación.

- Las transacciones entre oferta y demanda relativas al cargo por confiabilidad son transacciones del Mercado de Energía Mayorista (MEM).

- La OEF que se asigna a un generador resulta de una subasta transparente que ordena adelantar la Comisión de Regulación de Energía y Gas. De acuerdo con lo dispuesto en la Resolución CREG 071 de 2006, la valoración de la firmeza de una planta o unidad de generación se realiza en dos etapas, así: (i) primero, se determina la energía firme que es capaz de producir una planta para el cargo por confiabilidad y (ii) realizado lo anterior, se define la cantidad de energía firme que debe aportar una unidad o planta al sistema, de acuerdo con el despacho ideal, y se define igualmente el precio al que se pagará dicha firmeza o confiabilidad.

- La valoración de la energía firme que deberá aportar una unidad de generación al sistema (ENFICC), así como el precio al que se pagará dicha firmeza, se determinan a través de una subasta, de la que resulta la obligación de energía firme asignada a un generador y el derecho de este a ser remunerado, como contraprestación a la oferta por la cantidad de energía firme subastada. Sobra anotar que dicha remuneración es y ha sido 
pagada hasta la fecha por toda la demanda energética nacional en sus facturas, es decir, por los usuarios finales residenciales o no de dicha energía.

- La OEF es una opción financiera que encuentra respaldo físico en un recurso de generación que tiene la capacidad de producir la energía firme en momentos de escasez. La exigibilidad de la OEF se presentará cuando el precio de la energía en la Bolsa supere el precio de escasez (PE) determinado por el regulador. Dicho precio de escasez es (i) el referente regulatorio de la condición de estrés del sistema, y (ii) el valor que se le pagará al generador beneficiario de la OEF, en caso que este sea llamado a despachar su energía.

- Muy importante es anotar que habiendo recibido una OEF a través del mecanismo de subasta, la regulación no prevé que se requiera de ninguna formalidad adicional para vincular al generador y a la demanda al cumplimiento de la obligación de energía firme asignada y al pago del respectivo cargo por confiabilidad asociado a dicha obligación. Dado lo anterior, si el generador hace parte de forma voluntaria de un mercado cuyas reglas conoce, y adicionalmente ha recibido el pago asignado, en principio no sería posible alegar en el momento en que se requiere hacer efectiva su OEF, una indisponibilidad de sus activos, pues ello podría considerarse como un incumplimiento de la regulación vigente y, en la práctica, como un timo al mercado. En torno a este punto, la Resolución CREG 071 de 2006 prevé que si la indisponibilidad de un activo de generación es injustificada, y la indisponibilidad generó racionamiento, el respectivo agente deberá hacerse responsable de los perjuicios ocasionados por dicha situación, sin perjuicio de las demás sanciones que pueda imponer la Superintendencia de Servicios Públicos, en ejercicio de sus funciones.

En relación con lo expuesto, consideramos conveniente tener en cuenta que de acuerdo al artículo 33 de la Resolución CREG 71 de $2006^{22}$ «(...) las Obligaciones de Energía Firme que se asignen a cada generador tendrán el Período de Vigencia que esté definido en las normas que rigieron la realización de la Subasta, durante el cual se pagará la correspondiente remuneración prevista en esas mismas normas, sin perjuicio de los casos de incumplimiento por parte del generador previstos en la regulación, que le afecten la asignación y su remuneración".

- La liquidación, recaudo y pago del cargo por confiabilidad se efectúa a través de la Bolsa de Energía, por parte del Administrador del Sistema de Intercambios Comerciales (ASIC).

De otra parte, debe indicarse que la citada Resolución CREG 071 de 2006 definió la obligación de energía firme como ${ }^{23}$ :

"Obligación de Energía Firme: Vínculo resultante de la Subasta o del mecanismo que baga sus veces, que impone a un generador el deber de generar, de acuerdo con el Despacbo Ideal, una cantidad diaria de energía durante el Período de Vigencia de la Obligación, cuando el Precio de Bolsa supere el Precio de Escasez. Esta cantidad de energía corresponde a la programación de generación boraria resultante del Despacho Ideal basta una cantidad

22 Colombia, Comisión de Regulación de Energía y Gas - CREG. Op. cit.

23 Colombia, Comisión de Regulación de Energía y Gas - CREG. Op. cit. 
igual a la asignación becha en la Subasta, considerando solamente la Demanda Doméstica, calculada de acuerdo con lo definido en esta resolución" (subrayas fuera de texto).

Dado lo anterior, se tiene que la OEF es un deber que asume un generador por el hecho de participar en una subasta o en el mecanismo en que se le hace la respectiva asignación. Dicha obligación de energía firme nace a la vida jurídica como producto de una subasta en la que participan los generadores, a sabiendas de que asignada la OEF, ellos tendrán derecho a una remuneración por su confiabilidad, a cambio de la cual deberán generar, de acuerdo con el despacho ideal, una cantidad diaria de energía durante el período de vigencia de la obligación, únicamente cuando el precio de bolsa supere el precio de escasez.

Es así como, el artículo 52 de la citada resolución, vigente por demás, indica que:

"Las obligaciones de energía firme serán exigibles a cada uno de los generadores remunerados por concepto de Cargo por Confiabilidad durante cada una de las boras en las que el Precio de Bolsa sea mayor que el Precio de Escasez vigente. Dichas obligaciones deberán ser cumplidas de conformidad con el Despacho Ideal" (subrayas fuera de texto).

En cuanto al periodo de vigencia de la obligación, la resolución lo define como el "periodo de tiempo durante el cual un agente generador queda vinculado al cumplimiento de su Obligación de Energía Firme". Dicho periodo puede ser hasta de veinte años, según elija el generador con planta nueva, o hasta de diez años, según la elección del generador con planta especial, como se establece en el artículo 5 de la ya citada resolución.

De acuerdo con lo hasta aquí expuesto, se tiene que cuando un generador de forma libre se aviene a las reglas del mercado y de la metodología del cargo por confiabilidad, nace un deber para él que se denomina obligación de energía firme, que le impone entregar la energía asociada a la obligación asignada, de acuerdo con el despacho ideal, y la demanda del Sistema Interconectado Nacional, a cambio de una remuneración que es pagada puntualmente por todos los usuarios en sus facturas.

Valga la pena anotar que en sistemas eléctricos como el colombiano, en donde aproximadamente el $70 \%$ de la demanda es atendida de manera usual por centrales hidroeléctricas que dependen de unas determinadas condiciones hidrológicas, es técnica, económica y jurídicamente aconsejable que exista una oferta de generación total en términos de capacidad, superior a la demanda que debe ser atendida, de manera que ante condiciones críticas para las centrales hidroeléctricas, las centrales de respaldo (térmicas o no) puedan entrar a suplir la menor generación de las primeras, garantizando, en todos los casos, la prestación eficiente, continua e ininterrumpida del servicio para todos los usuarios.

Lo anterior conduce a que sea necesario el establecimiento de mecanismos como el del cargo por confiabilidad, que incentiven el desarrollo de proyectos que aporten capacidad de generación excedentaria al sistema, y a que implementados dichos mecanismos y en condiciones normales de hidrología, existan centrales que no sean despachadas en razón a sus costos marginales de generación, constituyendo dichas plantas unos activos 
de respaldo que, en todo caso, deben ser pagados por la demanda por su disponibilidad de prestar el servicio y de aportar confiabilidad al Sistema Interconectado Nacional.

Dado el planteamiento anterior, se entiende al cargo por confiabilidad como un mecanismo que busca incentivar y remunerar el desarrollo de capacidad excedente de generación, que permitirá la atención futura de la demanda, sin importar el crecimiento de esta, garantizando para los inversionistas un pago eficiente por la garantía de cobertura que estos ofrecen.

Sin embargo, y como dicha garantía no puede ser teórica, se impone a los inversionistas una obligación de generar energía en determinadas condiciones de precio, de manera que producida la condición que activa la obligación, esta pueda hacerse exigible en beneficio del sistema y de sus usuarios.

Desde este punto de vista, algunos podrían entender, y en efecto entienden, el esquema de cargo por confiabilidad como una forma atípica de contrato de seguro ${ }^{24}$, en donde el cargo mismo sería una prima, los usuarios que pagan el cargo serían los asegurados y las centrales beneficiarias del cargo se comportarían como aseguradores.

Bajo una perspectiva como la anotada, se tendría que verificado el riesgo que activa el seguro, el asegurador - generador debería cumplir con su obligación, sin importar el coste de esta, pues es así como tradicionalmente funciona el mercado del aseguramiento de riesgos.

No obstante la anterior posición, que sin duda ha hecho carrera en los medios de comunicación y en la opinión pública, para quienes presentamos este escrito, la conclusión que subyace a aquella es errada, pues a diferencia de lo que ocurre en un contrato de seguros típico, en el caso del cargo por confiabilidad, el hecho que activa la obligación de aseguramiento del riesgo es de muy probable ocurrencia (condiciones climáticas cíclicas). Por otra parte, no pueden igualarse las cargas de las compañías de seguros con las de las empresas generadoras de energía, pues estas últimas no cuentan con reaseguradoras a las que puedan acudir para mitigar sus riesgos. Estas, para desarrollar su actividad, dependen de unas estructuras de costos que son en extremo sensibles, y no hacen parte del sector financiero, sino en estricto sentido, del sector de infraestructura, lo que les impone que las actividades que desarrollen deban ser remuneradas de manera tal que no se vulnere el principio de suficiencia financiera a que se refieren las leyes 142 y 143 de 1994 .

De igual forma, debe tenerse en cuenta que a diferencia de los contratos de seguros, en donde le es posible al asegurador excusarse del cumplimiento de su obligación cuando, dada la circunstancia que activa el seguro, se presentan ciertas circunstancias imputables o no al asegurado (posición de riesgo) o a terceros, en el caso de los generadores beneficiarios del cargo por confiabilidad, no existen mecanismos que permitan a estos no concurrir a su deber de generar, toda vez que la condición de exigibilidad de la obligación es totalmente objetiva.

24 Finanzas. 'Ya pagamos por el seguro contra la escasez de energía'. Portafolio [en línea], 16 de octubre de 2015. Disponible en Internet: http://www.portafolio.co/economia/finanzas/pagamos-seguro-escasezenergia-24220 
Desde este punto de vista, insistimos en que el cargo por confiabilidad debe entenderse como una opción financiera que una vez activada, genera el deber de despachar una energía a un precio determinado. Dicha opción, en virtud del principio de suficiencia financiera a que se refiere la Ley 142 de 1994, debería estar acotada por los costos eficientes de generación de una planta en el momento de exigibilidad de la obligación de generar. Con una perspectiva como la anotada, y en situaciones en que el precio de bolsa supere al de escasez, los generadores con obligaciones de energía firme deberían entrar a generar, sí y solo sí, los costes de generación son remunerados de forma eficiente y con unos índices de rentabilidad mínimos, o siempre que superados dichos costes, se garantice al generador la recuperación futura del valor excedentario entre el coste real de generación y el valor de referencia al que el generador se comprometió a generar, previa la implementación de un mecanismo de verificación.

No obstante lo anterior, y a pesar de la preminencia legal del principio de suficiencia financiera, la CREG ha obviado dicho principio, haciendo exigible las OEF sin importar si el coste de generación de las plantas beneficiarias del cargo es superior o no al precio de escasez. Dado lo expuesto, el cálculo del precio de escasez por parte de la Comisión adquiere una importancia mayúscula, por lo que un cálculo errado de dicho precio bien podría generar responsabilidades pecuniarias para la CREG, por lo que se denominaría una falla del regulador.

En este punto, consideramos importante anotar que las obligaciones de energía firme se establecen a partir de la energía firme para el cargo por confiabilidad (ENFICC), y que la ENFICC es la máxima energía eléctrica que es capaz de entregar una planta de generación continuamente, en condiciones de baja hidrología, en un período de un año.

Dicha ENFICC, en una planta térmica como las que tradicionalmente aportan capacidad de respaldo al sistema, depende, entre otros factores, de: (i) la capacidad efectiva neta de la planta, (ii) de su comportamiento histórico y (iii) de la disponibilidad de los combustibles requeridos para la generación.

Los primeros dos factores anotados son factores objetivos y conocidos que no varían significativamente en el tiempo, y que son susceptibles de ser calculados con alto grado de certeza. Sin embargo, en lo que tiene que ver con el tercer factor, que es la disponibilidad de combustibles, se tiene que este incorpora un alea que tiene que ver con el precio de los combustibles, que varía con el tiempo, con el tipo de combustible que se emplee para generar, con la eficiencia técnica de la planta, con el inventario o stock de combustibles en un momento dado, con la capacidad de transporte con que cuente el país, e incluso con situaciones de orden público o de relaciones internacionales que puedan llegar a disminuir la cantidad de combustible requerida o que hagan que el valor de los combustibles supere la remuneración ficticia (precio de escasez) que la regulación ordena obtener por su uso.

Una vez se ha establecido la ENFICC de cada activo de generación, se realiza una asignación entre todas las plantas que tienen ENFICC con base en una demanda objetivo determinada por la CREG. Es así como la CREG obtiene la ENFICC asignada a cada planta. Desarrollado el proceso anterior, cuando el precio real de mercado de la energía en bol- 
sa supera el teórico precio de escasez que fija la CREG, surge la obligación de generar a ese precio de escasez, de acuerdo con el despacho ideal, sin exceder la ENFICC asignada.

Desde este punto de vista, se tiene que los generadores reciben el cargo por confiabilidad, dependiendo de su disponibilidad comercial, por lo que una planta declarada continuamente como indisponible no recibirá remuneración por concepto de dicho cargo. Por el contrario, en el caso de las plantas declaradas como disponibles, el cargo por confiabilidad se pagará independientemente de que la planta sea despachada o no, en la medida en que el cargo remunere la disponibilidad de la planta, así como la capacidad de generación de respaldo que esta provee.

Los agentes generadores que tienen cargo por confiabilidad o ENFICC asignada adquieren obligaciones de energía firme que, como se ha dicho, producen el deber de generar, de acuerdo con el despacho ideal, determinada cantidad de electricidad, siempre que el precio de bolsa supere el precio de escasez. Desde este punto de vista, la obligación de energía firme, además de ser un pago anticipado, se constituye en el vínculo jurídico que impone al generador un deber consistente en generar una cantidad de energía de acuerdo con un despacho ideal y hasta el límite de su ENFICC.

\section{PROBLEMÁTICAS REGULATORIAS ASOCIADAS AL DISEÑO DEL CARGO POR CONFIABILIDAD}

Como puede entreverse, el cargo por confiabilidad tiene un primer problema de diseño, el cual es la posible contradicción de su estructura con el principio de suficiencia financiera, lo cual puede llevar a que, en ocasiones, el coste de generar energía supere con amplitud los montos fijados como precio de escasez, haciendo insostenible, desde el punto de vista económico, la operación de una planta.

Dicho lo anterior, y focalizando el problema, se tiene que este tiene que ver con la forma y magnitud del precio de escasez que fija el regulador. En esa medida, y partiendo de la base de que, jurídicamente, el cargo por confiabilidad constituye una opción financiera cuyo precio de ejercicio debería ser reajustable conforme varíe el valor del bien subyacente, nos concentraremos ahora en el análisis económico del cargo y en la metodología que usa la CREG para fijar el precio de escasez.

Es así como, desde un punto de vista financiero y económico, podríamos señalar, como ya lo indicamos, que el cargo por confiabilidad se asemeja y es en realidad una opción financiera. Dichas opciones son instrumentos financieros derivados, que se constituyen en productos cuyo valor depende del precio de otro activo al que se denomina subyacente. En sus modalidades clásicas, las opciones financieras le dan al comprador el derecho, mas no la obligación, de comprar o vender un determinado bien (en este caso la energía, la cual es el activo subyacente) a un precio determinado, denominado strike o precio de ejercicio (que en este caso sería el precio de escasez), hasta una fecha concreta en la que vence la obligación.

Esas opciones pueden ser de compra, también denominadas call, o de venta, conocidas como put. En el caso del cargo por confiabilidad, estaríamos frente a una opción 
de compra o tipo call, en donde el subyacente o derivado sería la energía eléctrica, la remuneración del comprador de la opción o beneficiario del cargo sería el cargo por confiabilidad, y el strike o precio de ejercicio sería el precio de escasez.

Este precio de escasez, considerado como un precio de ejercicio, fue justificado por la CREG en el Documento CREG 043 de $2006^{25}$, el cual fue publicado para consulta el día 16 de junio de 2006 en la página web de la Comisión. En dicho documento, se definió el precio de escasez así:

"Dicho valor corresponde al precio al cual el generador se compromete a entregar la energía firme remunerada por concepto de Cargo por Confiabilidad, siempre que la opción sea ejercida, sin que intervenga el normal funcionamiento de la Bolsa. Este precio es el indicador de la condición crítica del sistema".

En la citada Resolución CREG 043 de 2006, se indicaron como objetivos principales del precio de escasez los de cubrir a la demanda contra precios altos, asegurar la entrega de energía adquirida mediante las obligaciones de energía firme y disminuir la incertidumbre en la estimación de los ingresos de los agentes generadores.

En lo que tiene que ver con la metodología de cálculo del precio de escasez, en la Resolución 043 citada, se establecieron las siguientes premisas: (i) el precio de escasez, denominado en el documento como precio de ejercicio, debe ser igual al valor de la planta térmica más costosa que pueda usar el sistema, de manera que se incentive el abastecimiento de la demanda para todos los recursos de generación disponibles (sistema marginalista); (ii) para lograr lo anterior, la CREG señala que debe considerarse el precio de la planta de menor eficiencia que pueda utilizar Fuel Oil n. ${ }^{\circ} 6$ como combustible alterno, asumiendo dicho combustible como el techo de la estimación del precio de ejercicio, y (iii) en materia de costos, deben tenerse en cuenta los de los combustibles referidos al mercado internacional, a los cuales deben sumarse los relacionados con la participación de los agentes en la bolsa de energía.

La fórmula de cálculo del precio de ejercicio puede ser consultada en el texto de la citada resolución, pero palabras más palabras menos, aquella responde a las premisas anotadas en el párrafo anterior, teniendo en cuenta, además, la eficiencia en el consumo de combustible de la planta de menor eficiencia, factor al que se denomina beat rate. Dicho factor de eficiencia es igual al consumo específico de combustible a plena carga de la planta menos eficiente que pueda utilizar el Fuel Oil n. ${ }^{\circ} 6$ como combustible alterno.

Para establecer el anterior y los demás factores de la fórmula, la CREG ordenó a las plantas térmicas que le reportaran información en función de su eficiencia, concluyendo

25 Colombia, Comisión de Regulación de Energía y Gas - CREG. Resolución CREG 043 de 2006 (17 de julio de 2006). Por la cual se ordena hacer público un proyecto de resolución de carácter general que pretende adoptar la CREG, con el fin de establecer la metodología para la remuneración del cargo por confiabilidad en el Mercado Mayorista de Energía. Publicada en el Diario Oficial n. ${ }^{\circ} 46.348$ el día 2 de agosto de 2006 . 
que la planta Barranca 3 era la más ineficiente, en el grupo de las que usaban Fuel Oil n. ${ }^{\circ}$ 6 , al tener un beat rate de $12.482 \mathrm{MBTU} / \mathrm{MWh}$ a plena carga.

En cuanto a la elección del Fuel Oil n. ${ }^{\circ} 6$ como combustible de referencia, la CREG lo justificó en la correlación verificable para dicha fecha, entre el comportamiento del precio en Bolsa de la electricidad frente al de dicho combustible.

La estructura de cálculo antes anotada fue la que finalmente se plasmó en la Resolución CREG 071 de 2006, por medio de la cual se establece la metodología del cargo por confiabilidad.

En términos generales, la metodología planteada parece lógica, pues al fijar como valor del precio de escasez el de la planta térmica más costosa que pueda usar el sistema, se garantiza, en teoría, que todos los recursos de generación disponibles, incluido el último en orden de eficiencia, puedan ver remunerados los costos de su generación sin pérdida alguna. Bajo ese contexto, y en el entendido de que, por regla general, siempre habrá más recursos de generación que demanda de estos, se presentaría un escenario ideal marginalista, en donde los costos de generación siempre estarían remunerados y en donde bajo ninguna circunstancia se estaría frente a la posibilidad de un racionamiento.

Lo anterior, teniendo en cuenta que un escenario de racionamiento eléctrico sólo podría presentarse cuando los recursos de generación sean inferiores a la demanda total del sistema, o cuando existiendo recursos de generación suficientes, el precio de generar sea superior a aquel que la demanda está dispuesta a pagar para obtener el servicio. Este último supuesto depende, como es lógico entenderlo, de la elasticidad precio - demanda de la energía.

No obstante lo expuesto, en la práctica, existen análisis que indican que el precio de escasez no remunera de forma completa los costos de generación de todas las plantas, pues en la fórmula solo se consideran los costos de generar con el Fuel Oil n. ${ }^{\circ} 6$ y los de participar en la Bolsa de Energía, pasando por alto todos los costos adicionales en que incurre una planta para estar disponible, además de los costos variables que estas puedan tener, así como el hecho de que los combustibles usados para generar tienden a variar con el tiempo.

De otra parte, señalan dichos estudios que al fijar como punto de referencia el valor de generación de una planta en específico, se pasan por alto las condiciones de eficiencia técnica propias de cada planta, así como las condiciones de contratación que estas tienen en cuanto al suministro de los combustibles requeridos para generar.

En relación con lo expuesto, la Asociación de Generadores de Energía (ANDEG) señaló en su Documento de Análisis n. ${ }^{\circ}$ 001-2014 ${ }^{26}$ lo siguiente:

"Desglosando los componentes del precio de oferta se analizaron los precios de los combustibles Fuel oil n. ${ }^{\circ}$, $1 \%$ y Fuel Oil n. ${ }^{\circ}$ 2, encontrando una diferencia promedio de 5.04 USD/MBTU. Esta diferencia es fundamental al momento de seleccionar el combustible referente en la valoración del precio de escasez ya que no se está consi- 
derando la planta más costosa del sistema ocasionando pérdidas considerables de energía en firme y eliminando los incentivos de generación para algunas tecnologías.

\section{GRÁFICO 7. DIFERENCIA PRECIOS COMBUSTIBLE}

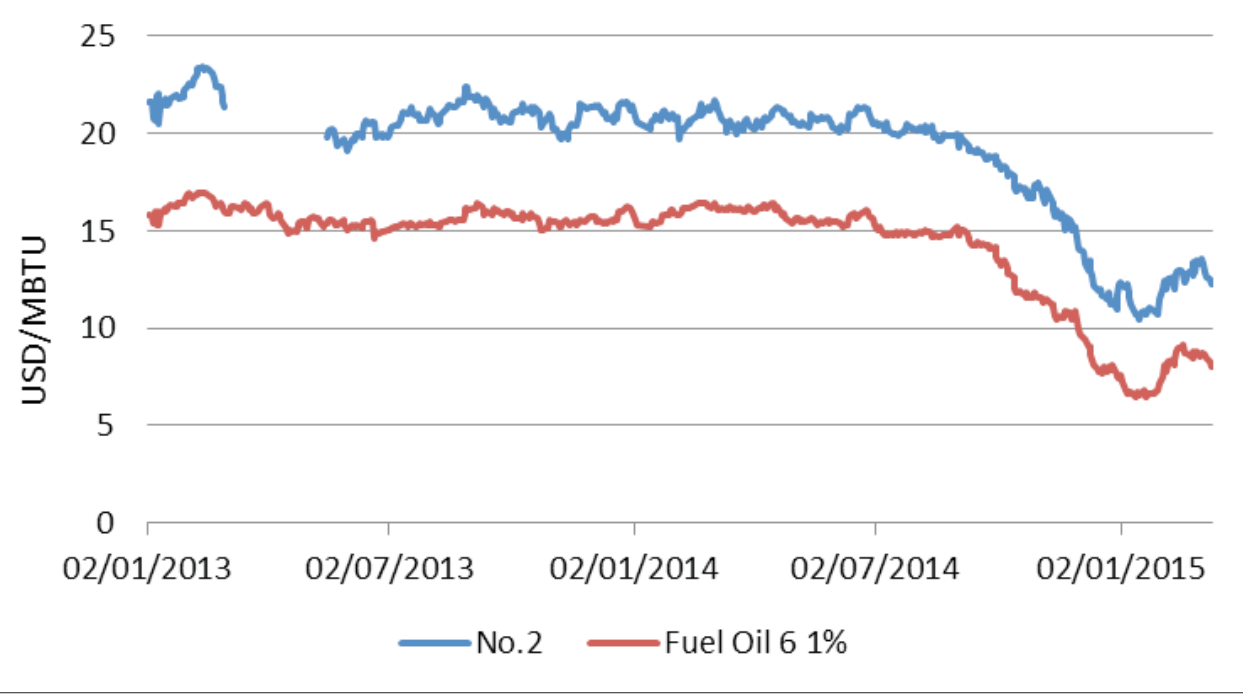

Respecto al 2015 analizando la curva de oferta de energía estimada del mes de febrero se evidencia que el precio de escasez revela rezagos en las señales de precio a causa de la metodología de cálculo vigente, generando mayores pérdidas económicas en aquellos meses en los cuales los precios de los combustibles presentan cambios significativos. Este riesgo de precio se podría gestionar mediante derivados financieros, sin embargo, es importante tener en cuenta que la venta de combustible a grandes consumidores en Colombia se realiza a precios regulados y no a costo de oportunidad o de referente internacional, situación que dificulta la realización del cubrimiento financiero necesario para mitigar estos riesgos de precio. Esta última condición de compra de combustibles líquidos a precios regulados produce una problemática adicional, en la medida que el precio de escasez es muy sensible a variaciones de los marcadores internacionales, mientras que para las plantas térmicas los precios de compra de combustibles no se ajustan por cuenta de la existencia del fondo de estabilización de precios nacionales para el Fuel Oil n. 2 y la gasolina.

Como se evidencia en el gráfico 8, el aumento en los precios del Fuel Oil n. 6 experimentado en el mes de febrero al no verse reflejado en el precio de escasez inmediatamente, ocasiona que los precios de oferta de las plantas que generan con este combustible sean mayores que el precio de escasez, dejando por fuera plantas que generan con combustibles Fuel Oil n. 6 y n. 2 las cuales representan el 30\% de la capacidad de generación". 


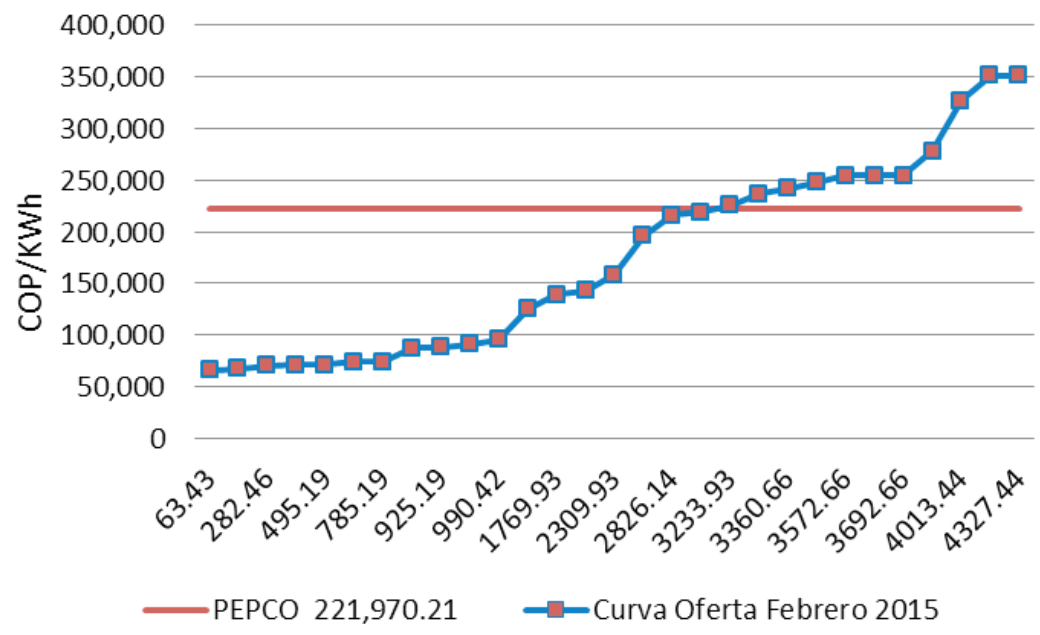

De acuerdo con el estudio de ANDEG, el cual debió y debería ser analizado por la CREG, la planta térmica más ineficiente capaz de usar Fuel Oil n. ${ }^{\circ} 6$ no es necesariamente la planta térmica más costosa del sistema, de lo que se deriva que en momentos en que la premisa no se cumpla, es decir, cuando existan plantas con costos de operación superiores a los de la última que emplea Fuel Oil n. ${ }^{\circ} 6$ como combustible de generación, se producirán pérdidas para dichas plantas que, eventualmente, tendrían que entrar a generar por debajo de sus costos cuando el precio de bolsa supere al de escasez.

Lo expuesto obliga a una revisión urgente de la metodología actual, pues como bien lo afirma ANDEG en el documento citado, "... la pérdida de la planta de generación impacta los ingresos de cargo por confiabilidad y por tanto la recuperación de los costos fijos asociados a la recuperación de la inversión en capital".

\section{PROBLEMÁTICA ACTUAL Y MECANISMOS DE SOLUCIÓN}

En la actualidad, y dado que se han presentado situaciones en las que algunas plantas térmicas llamadas a despachar no lo han hecho, a lo que se suma un atípico fenómeno de El Niño, así como fallas de algunas centrales de generación hidroeléctrica y térmica como Guatapé y Termoflores, respectivamente, el país se enfrenta a la amenaza de un racionamiento eléctrico. En torno al primero de los factores de amenaza anotados, el Comité de Seguimiento del Mercado de Energía Mayorista de la Superintendencia de Servicios Públicos Domiciliarios ${ }^{27}$ ha señalado, en su informe 104 de diciembre de 2015, que:

27 Comité de Seguimiento del Mercado de Energía Mayorista - CSMEM. Superintendencia de Servicios Públicos Domiciliarios. Informe 104 de 2015 [consultado el 29 de marzo de 2016] Disponible en Internet: http://www.superservicios.gov.co/MEM/archivos/informesexpert/informe104.pdf 
"En forma permanente las cantidades de generación térmica real ban sido inferiores a las programadas. Este desfase de generación trae como consecuencias: incremento de la generación bidráulica deteriorando las reservas bídricas, expone a los generadores bidráulicos a no poder cumplir con sus Obligaciones de Energía Firme futuras y la necesidad de mayores cantidades futuras de generación térmica. Las causas de la indisponibilidad de las plantas de generación térmica son principalmente fallas técnicas, falta de combustibles y pruebas de unidades. Bajo fallas de severidad importante están reportadas las plantas térmicas Cartagena 1, Paipa 1, Tasajero 2. También impactan la disponibilidad, los mantenimientos programados de plantas durante el primer trimestre del 2016. Es previsible y preocupante que los porcentajes de indisponibilidad de generadores térmicos por fallas y pruebas, se incrementen en el corto plazo, debido al estrés a que se someten las plantas, por la operación continua y con un alto factor de planta. Originalmente la mayoría de plantas térmicas del SIN fueron diseñadas para operar con gas natural y al baber sido modificadas para utilizar combustibles líquidos, su eficiencia disminuye y la probabilidad de falla se incrementa".

Ahora bien, en lo que tiene que ver con las indisponibilidades de los generadores térmicos, se tiene que estos han alegado de forma repetida, sustentada y de manera anterior a la crisis que hoy enfrenta el país, que el actual nivel del precio de escasez (PE) no está compensando los costos de generación térmica a partir de combustibles líquidos, lo cual tiene como causas, sin temor a decirlo, a: (i) la obstinación del regulador frente a la revisión de la metodología de cálculo del precio de escasez; (ii) una serie de factores externos imprevisibles, entre los que se cuentan un fenómeno de El Niño especialmente crítico y anormal, el cierre de la frontera con Venezuela y problemas de infraestructura de transporte, entre otros; (iii) una actitud propensa al riesgo por parte de algunos agentes que aceptaron recibir el cargo por confiabilidad en unas condiciones con las que no estaban de acuerdo, manteniendo prudencia en sus críticas en situaciones cómodas, y advirtiendo los problemas conocidos del esquema de manera pública ante situaciones críticas; (iv) un inadecuado cálculo de la Comisión de Regulación de Energía y Gas, quien, entre otros, fijó el valor del precio de escasez sin tener en cuenta la mejor información disponible de los agentes generadores y asumiendo que la planta más costosa no era la de mayor valor de generación, sino aquella más alta dentro de un grupo que le pareció apropiado, y (v) unas condiciones impositivas y de poder de mercado de los productores de combustibles, que no permiten a los generadores aprovechar la caída abrupta de los precios de los combustibles líquidos a nivel mundial.

Respecto de este último punto, si bien las variables con las cuales se calcula y actualiza mensualmente el precio de escasez (Fuel Oil International n. ${ }^{\circ}$ 6) ligadas al precio internacional del petróleo se encuentran deprimidas, el combustible líquido que requieren las térmicas no necesariamente es el Fuel Oil n. ${ }^{\circ} 6$, y tampoco deprimió su precio en iguales proporciones, dada su inmensa carga impositiva.

Este desbalance, que actualmente no solo pone en gravísimo riesgo a las generadoras térmicas con obligaciones de energía en firme a partir de líquidos, sino a todo el sistema eléctrico nacional a nivel financiero y técnico, debe ser compensado equilibradamente por todos los actores de la cadena (generadores, comercializadores, demanda y Estado), de manera que se lleve hasta un mínimo aceptable el riesgo de un racionamiento eléctrico. 
Frente a lo anterior, existen varias opciones:

1. No hacer nada. Bajo este escenario, los generadores térmicos podrían no llegar a cumplir con sus obligaciones de energía firme y sus compromisos de contratos de largo plazo, y la consecuencia de ello será que el sistema no pueda soportar la demanda diaria de electricidad, colapsando y llevándonos de forma necesaria a escenarios de racionamiento.

2. Compensar el desbalance a través de la modificación del precio de escasez, para que este sea más alto y remunere de mejor forma los costos involucrados en la generación de energía a través de plantas térmicas. Esta solución, si bien es lógica, generaría afectaciones en la demanda de usuarios finales de algunos pocos agentes comercializadores altamente expuestos a los precios de bolsa, los cuales sentirían un impacto muy alto en sus tarifas, lo que a su vez generaría un incremento a sus ya maltratadas carteras. Igualmente, una medida de este tipo llevaría a que los comercializadores se vean estresados financieramente por el aumento del valor de las garantías que deben constituir para transar en el mercado, lo que podría llevar a situaciones de incumplimiento de pagos y limitación de suministro.

3. A través de restricciones. En materia de energía eléctrica, la estructura tarifaria remunera las actividades de generación, transmisión, distribución y comercialización, además de los cargos relacionados con el cargo por confiabilidad, las pérdidas eficientes de energía reconocidas por el regulador a los agentes y las restricciones. Estas últimas son el mayor valor que debe pagar la demanda, como consecuencia de las restricciones eléctricas u operativas del SIN. Un escenario de aumento de las restricciones conduciría al incremento de tarifas a nivel nacional, lo que a su vez ocasionaría una subida en el valor de las garantías que tienen que entregar los comercializadores, como consecuencia del aumento del valor de la citada componente tarifaria.

El aumento indiscriminado de las garantías que son exigidas a los agentes para transar en el MEM podría llevar a posibles incumplimientos al mercado y de contratos de largo plazo por parte de muchos comercializadores, lo que llevaría a la quiebra de muchos de ellos, como ocurrió en El Niño de 2009, solo que en esta oportunidad, con una culpa que adecuadamente sustentada, bien podría imputarse al Estado colombiano por una falla del regulador.

Adicionalmente, la solución al problema vía aumento del valor de las restricciones conduciría al incremento de las tarifas de energía para todo el país, a lo que habría de sumarse el aumento de la inflación, las cargas impositivas, etc.

Este escenario también llevaría al incremento en la cartera de las empresas, a eventuales incumplimientos al mercado y a la reducción de la libre competencia en el mercado no regulado, dado que puede haber agentes que bajo dichas condiciones no estén interesados en atender demanda final.

Frente a las anteriores opciones, ha de decirse que el actual esquema de mercado, en especial la metodología del cargo por confiabilidad, fue diseñado teniendo en cuenta escenarios medios, optimistas y pesimistas. No obstante, las condiciones actuales son 
muy complejas para el sector energético: alto precio del dólar; alto precio de los combustibles a nivel nacional, a pesar de los bajos precios internacionales del petróleo; insuficiente oferta de gas natural, a lo que se suma una incomprensible política regulatoria de incentivo a la generación con gas, a pesar de que las reservas nacionales de dicho combustible a duras penas servirán para soportar la demanda residencial del combustible para los próximos años; déficit comercial; cierre de la frontera con Venezuela, que impide la llegada de líquidos importados; atraso de proyectos; incumplimiento de los compromisos de Venezuela en el suministro de gas a Colombia, y un fenómeno de El Niño fuerte, todos estos hechos que conducen a la necesidad de adopción de medidas extremas de intervención por parte del Gobierno Nacional para pasar esta crisis.

De esta forma, todos debemos hacer parte de la solución en proporciones equilibradas, por lo que proponemos se estudien las siguientes medidas:

1. En épocas de El Niño deben existir políticas del Estado colombiano dirigidas a "subsidiar" combustibles con destino a generación eléctrica, reduciendo el gasto en otros sectores, eliminando temporalmente las sobretasas e impuestos a los combustibles destinados a generación y redireccionando, a través de ECOPETROL y otras productoras, la entrega de líquidos a las plantas en condiciones de operar. Lo anterior exige, como es lógico entenderlo, la implementación de controles de precios de combustibles en épocas de estrés del sector eléctrico.

2. Debe considerarse que, de manera permanente, en períodos de verano se modifique la hora nacional, para que tal como ocurre en muchos países del mundo, se aproveche de mejor manera la luz solar y se reduzcan los consumos de energía. Respecto de este punto, las acciones deben ser permanentes y no sujetas a las eventualidades climáticas y a su severidad.

3. Debe modificarse la metodología de cálculo del precio de escasez, teniendo en cuenta las condiciones actuales, sin que se tenga un $100 \%$ de afectación del $100 \%$ a la demanda expuesta. Modificar el precio de escasez debe ser un acto de reconocimiento de la CREG de una falla en su regulación, así como de la necesidad de actualizar dicho precio a variables que, en la actualidad, son diferentes a aquellas que fueron diseñadas al momento de producirse la metodología del cargo por confiabilidad.

4. Debe reconocerse que el cargo por confiabilidad, si bien es una excelente herramienta para incentivar inversiones sectoriales y para aumentar la capacidad instalada de generación del país, tiene errores de estructura que contradicen el principio de suficiencia financiera establecido en la Ley 142 de 1994. Desde este punto de vista, debe modificarse la metodología del cargo por confiabilidad, reconociendo que este es un pago anticipado de la infraestructura que requiere el país para atender sus demandas energéticas, y no un pago anticipado de una energía que, como cualquier bien de mercado, sujeta su precio a las variables de oferta y demanda y a las estructuras de costos que existan en un momento determinado. Desde ese punto de vista, si bien es plausible que se fije un precio de escasez que sirva como detonante de la obligación de generar, deben establecerse herramientas que permitan a los generadores remunerar sus costos 
en desarrollo del principio de suficiencia financiera, propendiendo además por que en situaciones críticas, la demanda pague el valor real de generar, pero sin que se afecte de manera injustificada o sorpresiva.

Desde este punto de vista, la Superintendencia de Servicios Públicos Domiciliarios está en mora de establecer sistemas de medición de la eficiencia en la gestión de los generadores beneficiarios del cargo, que impidan que los costos de una gestión ineficiente se trasladen a los usuarios, y la CREG debe diseñar metodologías que permitan que los mayores costos eficientes que se generen en una situación de estrés del sistema sean asumidos de forma gradual por los usuarios, de manera que dichos valores se repartan en el tiempo.

En relación con este punto, y dado que el cargo por confiabilidad hace parte de la estructura tarifaria del servicio de energía, sería absolutamente comprensible que la Superintendencia de Servicios Públicos pudiese vigilar el uso de los recursos destinados a la garantía de confiabilidad, aun en relación con agentes que no se encuentren en etapas operativas. En este caso, no se vigilaría la prestación de un servicio, sino que se haría control tarifario de uno de los componentes de la fórmula tarifaria del servicio de energía, que efectivamente se estaría pagando en el momento de la vigilancia por parte de los usuarios.

5. La demanda necesariamente debe pagar un sobrecosto a través de un incremento de las restricciones. Sin embargo, dicho incremento debe ser limitado, de manera que cada uno de los componentes de la tarifa revele lo que en realidad cuesta llevar un kilovatio de energía a cada usuario conectado al SIN.

6. Los comercializadores deben mejorar su gestión de cartera y financiera, con el fin de cumplir a cabalidad sus obligaciones con el sector. En este punto, sería importante que tal como ocurrió en las primeras fases de nuestra regulación eléctrica, la CREG limite la cantidad de energía que pueden adquirir los comercializadores en la bolsa, cuando esta tenga por destino la atención del mercado regulado. Lo anterior, por cuanto al aumentar la cantidad de energía adquirida mediante contratos, se disminuye el riesgo de volatilidades extremas de la bolsa que afecten de manera inesperada a los usuarios.

De igual forma, vale la pena que la CREG corrija los errores que se encuentran en el modelo de Mercado Organizado (MOR) y expida las resoluciones que adopten dicho modelo, el cual vale la pena anotar, cumplirá este año 10 años en fase de discusión, es decir, todo un récord para un modelo de regulación.

7. Se deben desarrollar campañas efectivas y duraderas en el tiempo de concientización con los usuarios, para que estos conozcan la importancia de reducir sus consumos de energía en términos medioambientales, de eficiencia en el desarrollo de sus actividades y de reducción de tarifas. Ello puede ser especialmente útil, en tratándose de usuarios comerciales e industriales, quienes, en horas pico, podrían ayudar al sistema o entrar a generar, cuando ello sea posible, a través de las unidades de generación con las que cuenten.

En el caso de usuarios residenciales, las campañas deben ser sostenidas en el tiempo, y no concentradas en épocas de estrés del sistema, teniendo en cuenta que la energía embalsada que se ahorre en épocas de vacas gordas bien puede ser la que se requiera para encender una bombilla en épocas de vacas o embalses flacos. 
Vale la pena, en este punto, que la Comisión de Regulación de Energía y Gas tome el ejemplo de la CRA y expida resoluciones que castiguen de manera más efectiva el desperdicio de energía y/o incentiven la reducción de consumos en el tiempo.

8. Debe incentivarse de forma real el desarrollo de las fuentes no convencionales de generación de energía (FNCGE), respecto de las cuales el Gobierno Nacional está en mora de reglamentar de manera real la Ley 1715 de 2014. De igual forma, debe analizarse la conveniencia de seguir adelante con lo dispuesto en la resolución CREG 138 de 2015, que claramente desincentiva este tipo de desarrollos, los cuales pueden llegar a aportar energía firme al sistema, en condiciones como las presentes.

Respecto a este punto, parece inexplicable que mientras el mundo entero gira hacia la generación con este tipo de fuentes, la CREG siga considerando que este tipo de desarrollos desincentivan los grandes proyectos, sin detenerse a pensar que cada vez es más difícil desarrollar grandes proyectos hidroeléctricos, por los efectos ambientales que estos tienen, o térmicos, por los cuestionamientos ecológicos relacionados con las emisiones de carbono que este tipo de tecnologías generan.

En el caso de los pequeños aprovechamientos hidroenergéticos, definidos por la Ley 697 de 2001 como la energía potencial de un caudal hidráulico en un salto determinado que no supere el equivalente a los $10 \mathrm{MW}$, el legislativo debería analizar la posibilidad de ampliar dicho potencial hasta los $20 \mathrm{MW}$, que corresponde en la actualidad a las plantas no despachadas centralmente, o incluso de eliminar la restricción de potencial, siempre que se trate de plantas filo de agua (sin embalse), que demuestren, a través de estudios, que no están generando una desoptimización del potencial de las cuencas.

9. En el caso de empresas con alta exposición en bolsa, deben analizarse los casos particulares y coadyuvar a estas a contar con energía firme en contratos, para lo cual bien puede emplearse la posición cada vez más reducida que tiene el Estado colombiano en diferentes generadores, para forzar el suministro de energía a agentes que estén por encima de determinados índices de exposición en bolsa, con las penalidades e incentivos que se requieran.

10. En materia de vigilancia, la Superintendencia de Servicios Públicos Domiciliarios debe ser mucho más proactiva en la vigilancia de los agentes generadores y, aunque no viene al caso objeto de análisis, de los agentes transmisores. En relación con este punto, y desde hace varios años, se percibe una concentración de la Superintendencia en la atención de problemas asociados con la distribución y la comercialización de energía, que tienen un alto impacto mediático, pero que carecen de la potencialidad de resolver grandes problemas que podrían aliviar de una manera más efectiva las necesidades de los usuarios.

Lo anterior debe implicar un esfuerzo sostenido de tecnificación del ente de control, de manera tal que a ejemplo de otras entidades, como la Superintendencia de Industria y Comercio, sin descuidar los procesos de atención de peticiones, quejas y recursos (PQR), se realicen investigaciones efectivas que realmente coadyuven a solucionar los problemas que aquejan a los consumidores de servicios públicos. 
Al respecto de lo dicho, sorprenden en ocasiones los anuncios del ente de control cuando informa, por ejemplo, el inicio de investigaciones contra agentes como EPM por un eventual mantenimiento no realizado de la central hidroeléctrica Guatapé, pues tan reprochable puede ser el no realizar de manera presunta unas actividades de mantenimiento, como la conducta del ente de control, que sólo luego de muchos años y de un hecho de conocimiento nacional, se da cuenta de una situación que ha debido tener monitoreada desde hace mucho tiempo.

11. La Superintendencia debe iniciar de inmediato investigaciones preliminares a TODOs los agentes generadores, a efectos de establecer si han dado cumplimiento al Reglamento de Operación, si sus estructuras de costos son o no eficientes, si cuentan con la disponibilidad de los combustibles necesarios para operar en caso de que sean despachados, si han desarrollado los planes de mantenimiento requeridos para operar las plantas, si han honrado sus obligaciones de energía firme o, en el caso de que no hayan sido despachados, si están en condiciones de honrar dichas OEF. Estas investigaciones, una vez concluidas, deben llevar a la imposición de fuertes sanciones en caso de que se compruebe irresponsabilidad de los agentes, para lo que se propone que más que multas económicas altas, se piense en medidas como la separación del cargo de gerentes y administradores.

Adicionalmente, convendría que la Superintendencia de Servicios Públicos promueva una nueva modificación de la Ley 142 de 1994, que le permita, como sucede con la Superintendencia Financiera, participar en el proceso de selección de gerentes y administradores de empresas del sector eléctrico, de manera que se garantice que este sector no se maneje bajo criterios riesgosos y especulativos, que pueden llegar a depredar el mercado en situaciones de alta hidrología, y reclamar la acción salvadora del Estado en situaciones difíciles. Este análisis aplica igualmente para comercializadores altamente expuestos a la bolsa.

12. La CREG debe revisar si su actual política de incentivo a proyectos térmicos a gas es eficiente o no. Lo anterior, teniendo en cuenta que: (i) la disponibilidad de gas en Colombia es insuficiente, (ii) la seguridad en el suministro de energía no puede depender de la importación de un combustible y (iii) Colombia está asentada en una zona rica en carbón, con reservas que, eficientemente utilizadas, pueden durar cientos de años. En relación con este tema, debe abrirse la discusión con autoridades ambientales, productores y comunidad jurídica y económica, de manera que se incentive el desarrollo de proyectos de generación con carbón, con el menor impacto posible al medio ambiente.

13. Finalmente, consideramos que el Gobierno Nacional, el Congreso y la Academia deben analizar si existe o ha existido el riesgo de captura del regulador por parte de los generadores o del mercado en Colombia, si los generadores térmicos que incumplieron con sus OEF sabían que no podían cumplir con sus obligaciones y, en caso de que la respuesta a esta pregunta sea afirmativa, si debería darse un reintegro parcial de los recursos del cargo recibidos.

De acuerdo a lo expuesto, y aunque suene a frase de cajón, aquí todos tenemos que poner y perder, si no queremos perderlo todo. 
Para terminar, y superada la crisis, lo cual de seguro se hará, dada la fortaleza de nuestro sector eléctrico, debe evaluarse profundamente la regulación y pensar, con cabeza fría, si no ha llegado la hora de contar con un nuevo modelo de Comisión de Regulación de Energía y Gas, que renueve el sector, revalúe el esquema de cargo por confiabilidad, cierre procesos de discusión como el de la implementación del Mercado Organizado (MOR), que increíblemente está próximo a cumplir diez años en discusión, alinie el sector a las tendencias internacionales y lo refresque en cuanto a ideas, sin que ello implique un juicio a la labor de quienes, de manera responsable, a través de los últimos diez años, han ocupado la posición de comisionados.

\section{BIBLIOGRAFÍA}

Agudelo Villa, Hernando. De los monopolios a la democracia económica. Bogotá D.C.: Editorial Planeta Colombiana S.A., 1999.

ANDEG. Documento de análisis n. ${ }^{\circ}$ 01-2014, Un análisis del precio de escasez. ANDEG, 2014.

AréVAlo, Ismael HeRnANDo y otros. Derecho de los usuarios del servicio público de energía eléctrica y gas. Bogotá D.C.: Universidad Externado de Colombia, 2004.

Atehortua Rios, Carlos Alberto. Régimen de los servicios públicos domiciliarios en el contexto de la doctrina constitucional. Bogotá D.C.: Biblioteca Jurídica Dike, 2008.

Buby PIERRE y otros. Los servicios públicos en Europa. Hacia una regulación económica. Bogotá D.C.: Universidad Externado de Colombia, 2010.

Comité de Seguimiento del Mercado de Energía Mayorista - CSMEM. Superintendencia de Servicios Públicos Domiciliarios. Diferentes Informes [consultados el 29 de marzo de 2016]. Disponible en Internet: http://www.superservicios.gov.co/MEM/archivos/informesexpert/

Contraloría General de la República. Colombia: Entre la exclusión y el desarrollo - Propuestas para la transición al Estado Social del Derecho. Bogotá D.C.: CGR., 2012.

Gil Botero, EnRiQue. "La valoración jurídica de las manifestaciones normativas de las comisiones de regulación", en Revista Digital de Derecho Administrativo. 3. ${ }^{a}$ ed. Bogotá D.C.: Universidad Externado de Colombia, 2011.

Márquez Escobar, Carlos Pablo. Anotaciones sobre análisis económico del derecho. Bogotá D.C.: Pontificia Universidad Javeriana, 2005, vol 1.

Moreno CASTILLO, Luis FERnEy. Regulación del mercado de energía eléctrica en América Latina: La convergencia entre libre competencia e intervención estatal. Bogotá D.C.: Universidad Externado de Colombia, 2012.

SALAZAR, JORGE ENRIQUE. Simulación del cargo por confiabilidad y de la expansión del mercado de generación en Colombia. Tesis de máster. Escuela Técnica Superior de Ingeniería - ICAI, 2008.

SANTAElla QuinTERO, HÉCTOR. "Algunas reflexiones sobre las nuevas formas de actuación administrativa impuestas por el mercado y la técnica y sus implicaciones para la metodología de la ciencia jurídica administrativa", en Revista Digital de Derecho Administrativo. 5. a ed. Bogotá D.C.: Universidad Externado de Colombia, 2012.

XM S.A. E.S.P. ABC del cargo por confiabilidad. Disponible en Internet: http://www.xm.com.co/ Promocin\%20Primera\%20Subasta\%20de\%20Energa\%20Firme/abc2.pdf 